\title{
FORUM
}

\section{Nur wer jetzt sät, kann 2014 ernten - einige Vorschläge als Antwort auf die Beteiligung an den Europawahlen}

\author{
Eckart D. Stratenschulte*
}

Kaum ist der erste Schock der Europawahlen verdaut, setzen die relativierenden Analysen ein, die darauf hinweisen, dass in den ,alten ' EU-Ländern und in einigen ,neuen ‘ Mitgliedstaaten, wie beispielsweise Estland, die Wahlbeteiligung gestiegen sei. Diese Teilergebnisse sollen nicht bestritten werden, aber als Faktum bleibt, dass die Wahlbeteiligung insgesamt, wenn auch leicht gesunken ist. Hier gibt es nichts zu relativieren und schön zu reden. ${ }^{1}$ Nun könnte man die Leere in den Wahllokalen als gestiegene Zufriedenheit interpretieren, die dazu geführt haben könnte, dass die Bürgerinnen und Bürger Europas keinen Grund sahen, in das Geschehen einzugreifen. Aber: Wer in den letzten Monaten vor der Wahl an Diskussionen und anderen Veranstaltungen über Europa teilgenommen hat, weiß, dass dem nicht so ist. Die Wahlenthaltung ist vielmehr Ausdruck von Unmut und Unkenntnis über die Europäische Union, sie reflektiert Distanz und Entfremdung, die die Bürgerinnen und Bürger der Mitgliedstaaten gegenüber der Europäischen Union empfinden. Vor den Europawahlen gaben in einer Eurobarometerumfrage jeweils mehr als die Hälfte der Befragten an, dass ihre Stimme nichts ändere, dass sie nicht genug über das Europäische Parlament wüssten und sich für die Wahlen nicht interessierten, dass sie nicht ausreichend informiert seien, dass das Parlament sich nicht genügend um ihre Probleme kümmere und dass sie sich von den Europaabgeordneten nicht ausreichend vertreten fühlten. ${ }^{2}$

Internationale Organisationen können existieren, ohne dass die Menschen in den Mitgliedstaaten eine besondere Bindung an sie oder nur überhaupt relevantes Wissen über sie haben. Für die Europäische Union, die auf Supranationalität und Solidarität gründet und darüber hinaus fühlbar in unser tägliches Leben eingreift, gilt dies nicht. Sie bedarf der Akzeptanz der Bevölkerung und ihr größtes Lebensrisiko besteht im Verlust dieser Unterstützung, was nicht unbedingt zur Auflösung der Europäischen Union, aber zu ihrer Erosion führen würde.

Auch wenn längst andere Themen die politische Diskussion bestimmen, muss daher überlegt werden, wie man auf die unbefriedigende Situation, die sich in der Wahlbeteiligung gezeigt hat, reagieren kann. Einige Vorschläge seien hier skizziert:

* Prof. Dr. Eckart D. Stratenschulte, Leiter der Europäischen Akademie Berlin.

1 Ein Beispiel für die Relativierung der Bedeutung der Wahlbeteiligung gibt Daniel Gros vom Brüsseler ThinkTank CEPS, wenn er schreibt: ,The low level of participation has to be acknowledged, but it was actually better than predicted or could be expected given the lacklustre campaigns in most countries. It is particularly difficult to understand the extremely low participation rates (around 20\%) in some of the new member countries, even in Slovakia, which just joined the euro area, or the Czech Republic, which holds the rotating EU Presidency. But to see this EP election in a broader perspective: the abstention rate was about the same as in US mid-term Congressional elections." Daniel Gros: A more conservative Europe and EU!, CEPS Commentary, 08.06.2009, S. 2.

2 Europäische Kommission: Spezial Eurobarometer 299. Die Europawahlen 2009, September 2008, S. 18. 


\section{Mehr Einfluss}

\section{Personalisierung}

Die Kritik, die Bürger hätten in unserem Wahlsystem zu wenig Einfluss, da sie lediglich über von den Parteien vorgelegte und fixierte Listen abstimmen könnten, ist nicht neu. Bei den Europawahlen wird die eingeschränkte Wahlmöglichkeit objektiv dadurch verstärkt, dass es keine Erststimme zu vergeben gibt. Subjektiv wird der Eindruck, einer Liste zustimmen zu müssen, auf die man keinen Einfluss hatte, dadurch bekräftigt, dass den meisten Wählerinnen und Wählern die Kandidaten auf den verschiedenen Wahllisten unbekannt sind.

Eine Änderung des Wahlrechts auf Bundesebene könnte hier Abhilfe schaffen, indem ein Personalisierungselement eingebaut wird. Dies könnte wie in Österreich, den Niederlanden und ähnlich wie in einigen deutschen Kommunal- und Landeswahlrechten dadurch geschehen, dass man dem Wähler die Möglichkeit einräumt, Einfluss auf die Listenreihenfolge zu nehmen, beispielsweise durch die Abgabe einer, Vorzugsstimme ' für einen Kandidaten auf der Liste. ${ }^{3}$ Dies würde auch den Wahlkampf personalisieren. Falls den Wählern die Veränderung der Listenreihung möglich wäre, müssten auch die Frontmänner und -frauen kämpfen, da sie sich auf einen sicheren Listenplatz nicht verlassen könnten, und die Kandidaten auf den hinteren Plätzen hätten Chance und Anreiz. Dies würde zudem die Möglichkeit der Parteien, den Wahlkampf weitgehend mit Personal zu führen, das (wie die Kanzlerin oder der Außenminister) gar nicht zur Wahl steht, einschränken.

\section{Entscheidungsoptionen auf nationaler Ebene}

Eine weitere Kritik an den Wahlen zum Europäischen Parlament lautet, dass die Abstimmenden gar nicht wissen, wo eigentlich die Unterschiede zwischen den Parteien in europapolitischer Hinsicht liegen, dass sie also eigentlich nichts zu entscheiden hätten. Auch dieser Einwand ist alles andere als unbeachtlich. Tatsächlich stehen sich CDU und SPD europapolitisch viel näher als das bei der SPD und der britischen Labour Party der Fall ist, obwohl diese beiden zur selben politischen Familie gehören. Zwar wird im Wahlkampf - wenn er denn einmal stattfindet - durchaus auf die unterschiedlichen Positionen in Sachfragen hingewiesen, aber für die Wähler ist dies auch wegen der Komplexität der europäischen Entscheidungsfindung nicht nachvollziehbar. Es stehen eben anders als im Deutschen Bundestag nicht zwei Konzepte gegeneinander, von denen eines dann die Mehrheit erhält und mithin realisiert wird. Die Mehrheiten im Europäischen Parlament sind volatil und der Beschluss wird (bestenfalls) im Mitentscheidungsverfahren gefällt. Eine Möglichkeit, verschiedene Positionen deutlich und verstehbar zu machen, liegt auch hier in der Personalisierung der Wahl. Die Parteien sollten den Europawahlkampf mit ihrem Kandidaten für die Position des Kommissionsmitglieds bestreiten. Den Wählern könnten über die verschiedenen Kandidaten die unterschiedlichen Positionen besser verständlich werden. Eine solche Regelung ist ohne jede rechtliche Anpassung möglich. Die Parteien müssen lediglich ihre Kandidaten präsentieren und die Bundesregierung muss sich verpflichten, das Wählervotum bei der Benennung der entsprechenden Persönlichkeit zu respektieren.

3 Vgl. zum Beispiel: Bundesministerium für europäische und internationale Angelegenheiten der Republik Österreich: Informationen in Deutsch. EP Wahl 2009, abrufbar unter: http://www.bmeia.gv.at/botschaft/auslandsoesterreicher/ratgeber/wahlen/ep-wahl-2009/informationen-in-deutsch.html (letzter Zugriff: 12.06.2009); eine allgemeine Darstellung findet sich auch bei Martin Fehndrich: Vorzugsstimmen, in: Wilko Zicht/Martin Fehndrich/Matthias Cantow (Hrsg.): Wahlrechtslexikon, abrufbar unter: http://www.wahlrecht.de/lexikon/vorzugsstimmen.html (letzter Zugriff: 12.06.2009). 


\section{Entscheidungsoptionen auf europäischer Ebene}

Ein ähnlicher Prozess sollte auf europäischer Ebene dadurch stattfinden, dass die politischen Familien vor der Wahl ihre Kandidatin beziehungsweise ihren Kandidaten für das Amt des Kommissionspräsidenten benennen und mit ihr beziehungsweise ihm werben. Der SPD-Spitzenkandidat Martin Schulz erklärte während des Wahlkampfs, seine Gruppierung werde, falls sie die stärkste Kraft im Europäischen Parlament werde, die erneute Nominierung von José Manuel Barroso verhindern. Das alleine ist allerdings für den Prozess der Akzentuierung der Wahlentscheidung nicht hilfreich, solange kein eigener Kandidat präsentiert wird und der politische Mitbewerber die Aufforderung zum Tanz nicht annimmt. Zwar hatte die Europäische Volkspartei kurz vor den Europawahlen beschlossen, eine zweite Amtszeit von Präsident Barroso zu unterstützen, aber diese Personalie wurde im Wahlkampf nicht mit der Wahlentscheidung verknüpft, sodass die Wählerinnen und Wähler nicht das Gefühl haben konnten, auch über den Kommissionspräsidenten zu entscheiden.

Legten sich die Parteifamilien vorab auf eine Kandidatin oder einen Kandidaten für das Amt des Kommissionspräsidenten fest, würden sie zwangsläufig auch die Praxis der Staatsund Regierungschefs torpedieren, einen Kommissionspräsidenten nicht nach Stärke, sondern nach Schwäche auszusuchen.

Wenig hilfreich ist hingegen der Vorschlag, den Präsidenten des Europäischen Rates von der Bevölkerung der Mitgliedsländer direkt wählen zu lassen, wie dies Wolfgang Schäuble vorschlug. ${ }^{4}$ Dies würde eine vollständige Änderung des durch den Lissabonner Vertrag gerade (hoffentlich) gewonnenen Systems bedeuten. Es ist schwer vorstellbar, europaweit einen Präsidenten des Europäischen Rates zu wählen, ohne diesen dann mit weitreichenden Kompetenzen auszustatten. Eine solche Wahl könnte auch nicht für zweieinhalb Jahre vorgenommen werden. Würde man den Bürgern anbieten, den jetzt im Lissabonner Vertrag vorgesehenen Moderator zu wählen, führte dies zu zusätzlicher Frustration, da die Wähler das berechtigte Gefühl hätten, einen machtlosen Politiker bestimmen zu dürfen.

\section{Mehr Transparenz}

Die Intransparenz des europäischen Entscheidungssystems ist oft beklagt worden und zu einem nicht unerheblichen Teil auch nicht zu beseitigen. Politische Aushandlungsprozesse, die sich oft über Jahre erstrecken und zwischen 27 Staaten, verschiedenen politischen Familien und unterschiedlichen sozialen Interessen geführt werden, entwickeln nicht die Durchschaubarkeit, die man sich als politischer Zuschauer wünschen würde. Umso wichtiger ist es, das Maß an Transparenz zu gewährleisten, das möglich ist.

\section{Mehr Präsenz bei den Wählern}

Neben den modernen technischen Möglichkeiten wie dem Internet muss die Transparenz vor allem durch diejenigen hergestellt werden, die in diesen Politikprozess entscheidend eingebunden sind, die Europaparlamentarier. Diese allerdings verfügen kaum über Zeit, in den Wahlregionen präsent zu sein. Das Europäische Parlament führt jährlich circa 40 Plenarund Ausschusssitzungswochen durch, wodurch kaum Zeit für die Wahlkreisarbeit zu Hause bleibt. Zum Vergleich: Der Deutsche Bundestag kommt mit 22 Sitzungswochen aus. Dem Deutschen Bundestag gehören aktuell 612 Personen an, die Zahl der deutschen Europaparlamentarier liegt bei 99. Dies bedeutet: Ein Sechstel der Abgeordnetenzahl muss die Verbin-

4 dradio.de: Schäuble schlägt Direktwahl eines EU-Präsidenten vor, 09.06.2009, abrufbar unter: http://www.dradio.de/nachrichten/200906090600/6 (letzter Zugriff: 11.06.2009). 
dung zu den Wählern halten und hat dafür halb so viel Zeit. Das Europäische Parlament sollte daher die Zahl seiner Sitzungswochen deutlich reduzieren. Die intensivsten Beratungen in Ausschüssen und im Plenum helfen nichts, wenn dadurch die Verbindung zur Bevölkerung abreißt. Die Zahl der Sitzungswochen liegt in der Regelungskompetenz des Parlaments, die Abgeordneten können sich also in dieser Hinsicht am eigenen Schopf aus dem Sumpf ziehen.

\section{Schluss mit dem Wanderzirkus}

Eine Möglichkeit, Energie (und Kosten) zu sparen, ist die Abschaffung des offiziellen Sitzes des Europäischen Parlaments in Straßburg. Der Öffentlichkeit ist dieser Wanderzirkus sowieso nicht zu vermitteln und Frankreichs Einbindung in die Europäische Union ist auch nicht so schlecht, dass es des Bindeglieds der Plenarsitzungen in Straßburg bedürfte. Für die Parlamentarier würden durch die Abschaffung des ständigen Platzwechsels Kapazitäten frei, die sie für ihre sonstige Arbeit und den verstärkten Wählerkontakt gut nutzen könnten. Allerdings liegt diese Frage in der Kompetenz des Rates, der zudem einstimmig darüber entscheiden und somit den französischen Widerstand überwinden muss. Dennoch sollte dieses Vorhaben nunmehr angepackt werden. Die Zukunft der Europäischen Union kann auch dem französischen Präsidenten nicht gleichgültig sein.

\section{Bessere Ausstattung}

Wie viel ein Politiker erreichen kann, hängt auch stark davon ab, auf welche personellen Ressourcen er zurückgreifen kann. Diese sind bei den Europaparlamentariern äußerst begrenzt und beschränken sich in der Regel auf ein bis zwei Personen in Brüssel sowie ein bis zwei weitere zu Hause. Dieses Team muss für die Abgeordneten die gesamte Unterstützungsleistung von der Sichtung und Bewertung wissenschaftlicher Studien (, Wie gefährlich ist Acrylamid?') über die Sitzungsvorbereitung, die Organisation von Pressekonferenzen, die Flugbuchungen, den Kontakt mit den Wählern, Interessenverbänden und Besuchergruppen bis zu Chauffeurdiensten im Wahlkreis leisten. Zum Vergleich: Ein US-amerikanischer Kongressabgeordneter kann über 18 Vollzeit- und vier weitere Teilzeitmitarbeiter verfügen, die durchschnittliche Zahl der Abgeordnetenmitarbeiter liegt in den USA bei $15 .{ }^{5}$ Würden die Teams der Europaabgeordneten entsprechend vergrößert, wäre ihre Wirkungsmöglichkeit wesentlich verstärkt. Kostenmäßig würde sich eine solche Vergrößerung auf circa 46 Millionen Euro belaufen, also rund 0,03 Prozent des jetzigen EU-Budgets. So viel wie den kostenbewussten Amerikanern sollte den Europäern ihre Demokratie auch wert sein, das müsste auch den letztlich darüber entscheidenden Mitgliedstaaten akzeptabel erscheinen.

\section{Mehr Wahlkampf}

Wahlkämpfe sind für den demokratischen Entscheidungsprozess wichtig. Der Kampf der Parteien um Stimmen und die damit einhergehende Werbung führt zur einer starken Präsenz der Themen in der öffentlichen Diskussion und weckt - auch über Zuspitzungen und Polemik - Interesse an den Inhalten, die zur Wahl stehen, sowie am Wahlgang selbst. Dies setzt allerdings voraus, dass die Parteien diesen Wahlkampf auch wirklich führen. Zumindest in Deutschland war zu beobachten, dass die Parteien sich dem Wahlkampf verweigert haben, um - wie in Diskussionen von Politikern auch freimütig zugegeben wird - einen Teil der

5 Vgl. John S. Pontius/Faye M. Bullock: Congressional Staff: Duties and Functions, CRS Report for Congress, Order Code 98-340 GOV, Washington D.C. 2003. 
Wahlkampfkostenerstattung für den Bundestagswahlkampf zurückzuhalten. Tatsächlich werden also keine Kosten erstattet, sondern es wird vom Steuerzahler ein Bonus gewährt, der umso größer ausfällt, je weniger eine Partei der Idee folgt, die der Finanzhilfe zugrunde liegt. Hier ist Abhilfe durch eine einfache gesetzliche Änderung möglich, die besagt, dass nur Kosten erstattet werden, die im jeweiligen Wahlkampf tatsächlich entstanden sind und eine Querfinanzierung von Wahlkämpfen ausgeschlossen ist.

\section{Mehr Vorausschau}

Ein wichtiger Multiplikator für die politische Diskussion ist nach wie vor der Deutsche Bundestag, der europäische Themen im Plenum allerdings meistens erst aufnimmt, wenn es gilt, Richtlinien der Europäischen Union in nationales Recht umzusetzen. Dies markiert jedoch das Ende des europäischen Gesetzgebungsprozesses, an dem Änderungen zudem praktisch nicht mehr möglich sind. Wichtig wäre, dass der Deutsche Bundestag die politische Debatte um europäische Regelungen am Anfang des Verfahrens führt. Damit kann einerseits die Bundesregierung stärker gebunden werden, andererseits wird auch deutlich, dass in Europa um Entscheidungen gerungen wird und nicht über Nacht Regelungen aus Brüssel kommen, die wir in Deutschland übernehmen müssen. Nötig ist für eine solche Veränderung lediglich der Wille des Deutschen Bundestags.

Dass das Bundesverfassungsgericht mittlerweile in seinem Urteil zum Vertrag von Lissabon ${ }^{6}$ die stärkere Mitwirkung des Bundestages gefordert hat, kann in diesem Zusammenhang als Chance gesehen werden.

\section{Mehr Repräsentativität}

Vertraglich fixiert steht jedem Mitgliedstaat eine bestimmte Anzahl von Sitzen im Europäischen Parlament zu, dabei ist es unbeachtlich, wie hoch die Wahlbeteiligung im jeweiligen Staat ist. Diese Regelung treibt weder die Parteien besonders an, auf eine hohe Wahlbeteiligung hinzuwirken, noch schafft sie eine tatsächliche Repräsentativität der schließlich nach Brüssel und Straßburg entsandten Abgeordneten. Da erfahrungsgemäß die Unterstützer extremer Parteien an den Wahlen teilnehmen, werden ihre Vertreter anschließend im Parlament stärker vertreten sein als dies der politischen Einstellung der Bevölkerung, die sie im Parlament repräsentieren sollen, entspricht. Beides ist schwer einzusehen. Wenn tatsächlich 80 Prozent der Litauer der Ansicht sind, ihre Bevölkerung solle keinen Einfluss auf die Geschehnisse in Brüssel und Straßburg nehmen, warum sollen ihnen dann dennoch 100 Prozent ihres Sitzkontingents zustehen? Das Wahlrecht zum Europäischen Parlament sollte dahingehend geändert werden, dass jedem Staat unabhängig von der Wahlbeteiligung eine bestimmte, ungefähr 50 Prozent des Kontingents umfassende Sitzzahl zusteht, er das volle Kontingent jedoch nur stufenweise mit einer bestimmten Wahlbeteiligung erreichen kann. Vorstellbar wäre beispielsweise, dass die volle Sitzzahl nur in Anspruch genommen werden kann, wenn eine Wahlbeteiligung von 70 Prozent erreicht ist. Damit wird für die Wählerinnen und Wähler auch klar, dass sie neben der parteipolitischen Präferenz darüber entscheiden, ob sie überhaupt im Entscheidungsprozess repräsentiert sein wollen. Vermutlich dürfte eine solche Regelung zu einer wesentlichen Erhöhung der Wahlbeteiligung führen, um den eigenen Einfluss zu sichern. Auch die politischen Parteien müssten verstärkt nicht nur um Wähler, sondern um die Wahlbeteiligung werben, weil sonst, sichere Listenplätze ' nicht mehr mit einem Ticket nach Europa belohnt werden würden.

6 BVerfG, 2 BvE 2/08 vom 30.06.2009. 
Diese Forderung mag auf den ersten Blick unrealisierbar erscheinen. Tatsächlich setzt sie eine Vertragsänderung voraus, die nicht leicht erzielbar ist. Allerdings hat die Europäische Union mittlerweile viele Dinge verwirklicht oder angepackt, die man lange Zeit für unvorstellbar hielt, von der Währungsunion bis zur Gemeinsamen Verteidigungspolitik. Da müsste doch eine vergleichsweise kleine Vertragsänderung möglich sein, die niemandem Einfluss nimmt, sondern lediglich allen mehr Anstrengung im Interesse der gemeinsamen Sache abverlangt. So utopisch, wie die Forderung im ersten Augenblick klingt, ist sie übrigens nicht. Bei den Wahlen zum Deutschen Bundestag findet nämlich ebenfalls eine Korrelation zwischen Wahlbeteiligung und Repräsentation in Berlin statt. Wenn in einem Bundesland die Wahlbeteiligung niedriger als im Bundesdurchschnitt ist, ziehen aus diesem Land weniger Parlamentarier in das Reichstagsgebäude ein, ${ }^{7}$ da die Gesamtzahl der Mandate, die eine Partei errungen hat, nach dem jeweiligen Stimmenanteil in den Bundesländern vergeben wird.

\section{Mehr Bildung}

Einstellungen entwickeln sich langsam und sind durch kurzfristige Werbekampagnen nicht wirklich zu verändern. Das hat sich auch bei den Europawahlen wieder gezeigt. Die Akzeptanz der Europäischen Union und das Verständnis für sie lassen sich daher nur langfristig vergrößern, indem man ,Europa‘ zu einem selbstverständlichen Teil des politischen Diskurses macht.

\section{Europa als Unterrichtsprinzip}

Bereits 1978 haben die Kultusminister der Länder vereinbart, in den deutschen Schulen Europa zum Unterrichtsprinzip zu machen. Nicht nur, dass das Thema Europäische Union als Gegenstand behandelt werden soll, es ist vorgesehen, in allen Schulfächern die europäischen Bezüge herzustellen. In der Überarbeitung des Beschlusses vom 5. Mai 2008 heißt es: „Ziel der pädagogischen Arbeit an Schulen muss es sein, in den jungen Menschen das Bewusstsein einer europäischen Identität zu wecken und zu fördern. Hierzu gehört auch die Vorbereitung der jungen Menschen darauf, ihre Aufgaben als Bürgerinnen und Bürger in der Europäischen Union aktiv wahrzunehmen." ${ }^{8}$

Dem ist nichts hinzuzufügen, außer der Feststellung, dass die Empfehlungen der Kultusminister schon in den Lehrplänen nicht umgesetzt werden ${ }^{9}$ und das in der Unterrichtspraxis noch viel weniger der Fall ist.

Um diesem Zustand abzuhelfen, bedarf es keiner neuen rechtlichen Regelungen, sondern lediglich der Implementierung der längst gefassten Beschlüsse. Dies wird allerdings nicht möglich sein, ohne die ,europäische Bildung ' der Lehrer in Deutschland mit einem kräftigen Schub zu versehen und verstärkt neue Unterrichtsmaterialien zu entwickeln, die - auch beispielsweise im Sprachunterricht - tatsächlich Europa als Unterrichtsprinzip vorsehen.

7 Vgl. Art. 1 und Art. 6 Bundeswahlgesetz in der Fassung der Bekanntmachung vom 23. Juli 1993 (BGB1. I S. 1288, 1594), das zuletzt durch Artikel 1 G. vom 17. März 2008 (BGB1. I S. 394) geändert worden ist.

8 Ständige Konferenz der Kultusminister der Länder in der Bundesrepublik Deutschland: Europabildung in der Schule. Empfehlung der Ständigen Konferenz der Kultusminister der Länder in der Bundesrepublik Deutschland (Beschluss der Kultusministerkonferenz vom 08.06.1978 i. d. F. vom 05.05.2008), S. 5.

9 Vgl. Maja v. Geyr/Lilly Hornung/Friederieke Noack/Jaroslav Šonka/Eckart D. Stratenschulte/Lisa Moldenhauer: Die Europäische Dimension in den Lehrplänen der Bundesländer. Vergleichende Studie im Auftrag der Europäischen Kommission - Vertretung in Deutschland, herausgegeben und erarbeitet von der Europäischen Akademie Berlin, Berlin 2007. 


\section{Überzeugung statt Überwältigung}

Man kann den öffentlichen Stellen sowohl in der Europäischen Union als auch in der Bundesrepublik Deutschland nicht vorwerfen, dass sie das Thema Europäische Union nicht durch zahlreiche Materialien und Maßnahmen aufgreifen würden. Allerdings werden die meisten dieser Publikationen und Veranstaltungen nach den Grundsätzen der Öffentlichkeitsarbeit erstellt, in denen das Gute betont, das nicht so Gute uminterpretiert und das Problematische verschwiegen wird. Für die Bildung in Schule und Erwachsenenpädagogik eignen sich solche Maßnahmen nicht, da sie als Propaganda aufgefasst und abgetan werden. Für die politische Bildung in Schule und Gesellschaft gibt es klare Grundsätze, die einst im Beutelsbacher Konsens zusammengestellt wurden. Demzufolge dürfen der Schüler oder die Teilnehmerin nicht überwältigt werden, muss das in der Gesellschaft Kontroverse auch kontrovers dargestellt werden und muss den Rezipienten die Möglichkeit gegeben werden, eine politische Situation im Hinblick auf ihre eigene Interessenlage zu analysieren. ${ }^{10}$ Dies geht allerdings nur in dialogisch angelegten Veranstaltungen und mit Materialien, die der Leserin oder dem Nutzer eine Auseinandersetzung mit dem Thema ermöglichen. Neue rechtliche Regelungen oder zusätzliche Finanzmittel sind hier nicht vonnöten, es geht lediglich (aber auch immerhin) darum, das Vorhandene anders zu gestalten.

Die geringe Wahlbeteiligung zum Europäischen Parlament im Jahr 2009 stellt für sich genommen keine Katastrophe dar, sie ist aber ein Warnzeichen, das ernst genommen und auf das reagiert werden sollte.

10 Vgl. Hans-Georg Wehling: Konsens à la Beutelsbach? Nachlese zu einem Expertengespräch, in: Siegfried Schiele/Herbert Schneider (Hrsg.): Das Konsensproblem in der politischen Bildung, Stuttgart 1977, S. 173184, hier S. 178-180. Der Konsens wurde 1976 auf einer Tagung der Landeszentrale für politische Bildung Baden-Württemberg entwickelt und nach dem Tagungsort benannt. 